\title{
Dermoscopy of a single plaque on the face: an uncommon presentation of cutaneous sarcoidosis
}

\author{
Claudio Conforti ${ }^{1}$, Roberta Giuffrida ${ }^{2}$, Mayara Hamilko de Barros ${ }^{3}$, \\ Fernanda Simoes Seabra Resende ${ }^{4}$, Lorenzo Cerroni ${ }^{4}$, Iris Zalaudek ${ }^{1}$
}

\begin{abstract}
1 Dermatology Clinic, Maggiore Hospital, University of Trieste, Trieste, Italy
2 University of Messina, Department of Clinical and Experimental Medicine, Section of Dermatology, Messina, Italy

3 Charity Hospital of Rio de Janeiro, Institute of Dermatology, Rio de Janeiro, Brazil

4 Department of Dermatology, Medical University of Graz, Graz, Austria
\end{abstract}

Key words: plaque, cutaneous sarcoidosis, dermoscopy, inflammatory disease, face, granulomatous disease

Citation: Conforti C, Giuffrida R, Hamilko de Barros M, Resende FSS, Cerroni L, Zalaudek I. Dermoscopy of a single plaque on the face: an uncommon presentation of cutaneous sarcoidosis. Dermatol Pract Concept. 2018;8(3):174-176. DOI: https://doi.org/10.5826/ dpc.0803a04

Received: October 31, 2017; Accepted: March 1, 2018; Published: July 31, 2018

Copyright: $@ 2018$ Conforti et al. This is an open-access article distributed under the terms of the Creative Commons Attribution License, which permits unrestricted use, distribution, and reproduction in any medium, provided the original author and source are credited.

Funding: None.

Competing interests: The authors have no conflicts of interest to disclose.

All authors have contributed significantly to this publication.

Corresponding author: Claudio Conforti, MD, Università Campus Bio-Medico (UCBM), Via Alvaro del Portillo 200, 00128 Rome, Italy. Email: claudioconforti@yahoo.com.

ABSTRACT Sarcoidosis is a multisystemic granulomatous disease of unknown causes, and cutaneous sarcoidosis (CS) is an early manifestation of the disease. Dermoscopy has gained increasing interest in the past few years as an aid in the clinical diagnosis of inflammatory and infectious skin manifestations. We present a case report about a single, erythematous, and asymptomatic plaque on the face with unexpected dermoscopy characteristics of CS.

Learning points:

- CS on the face of a therapy-resistant actinic keratosis should be considered a differential diagnosis.

- Dermoscopy can change the diagnosis and lead to the correct management.

\section{Case Presentation}

A 43-year-old woman was referred to our skin cancer unit with a history of therapy-resistant actinic keratosis (AK), which had been treated by a practice-based dermatologist with topical isotretinoin and 3\% diclofenac in $2.5 \%$ hyaluronic acid without improvement. Clinically, the lesion appeared as a reddish, slightly scaling plaque $6 \mathrm{~mm}$ in diameter on the right temple (Figures 1 and 2). The patient recalled the presence of the lesion for the past several months but denied any symptoms or history of trauma.

The digital epiluminescence microscope system with polarized light (DermLite, 3Gen, $\times 20$ ) with immersion oil revealed whitish-yellow scales and whitish structureless areas on a yellow-orange background, with diffuse linear irregular vessels (Figure 2). Due to the yellow-to-orange color, which is typically seen in granulomatous diseases, the differential diagnosis included CS, lupus vulgaris (LV), and 


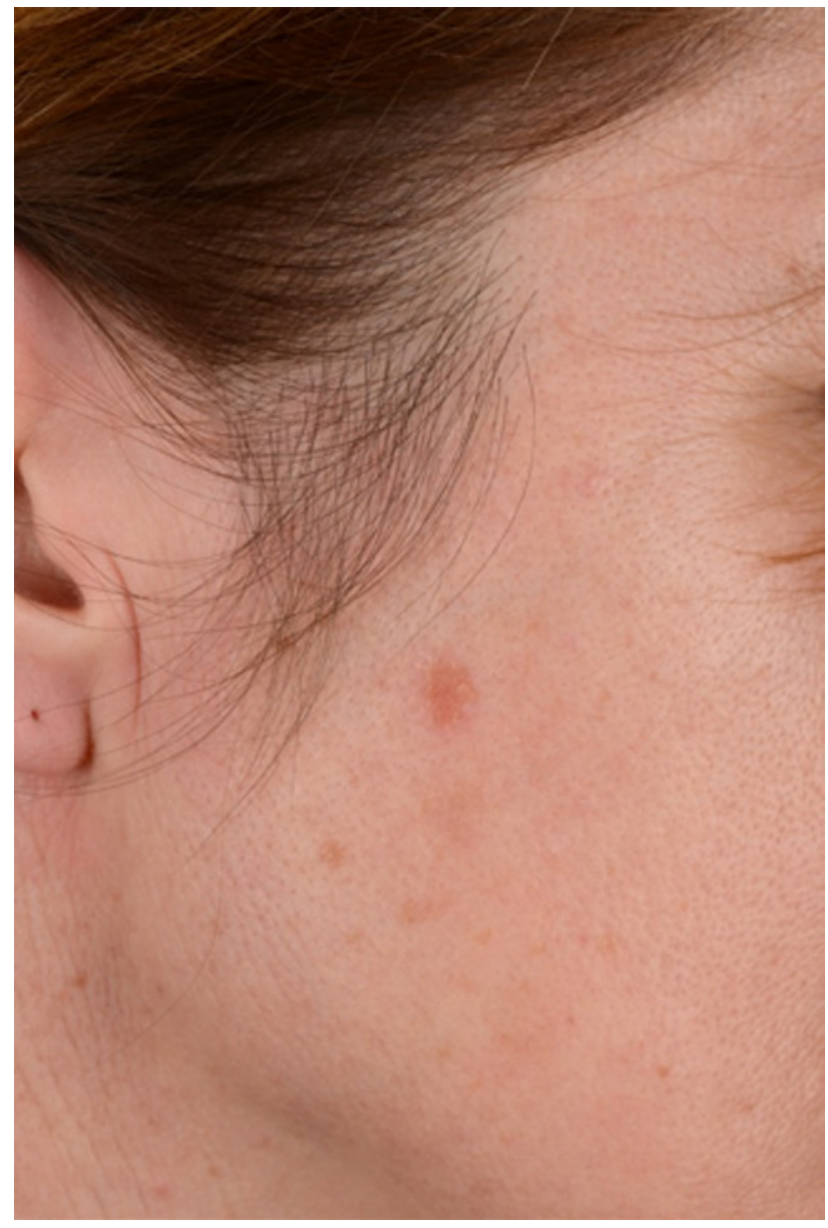

Figure 1. Clinical image of a single erythematous plaque on temple area. [Copyright: @2018 Conforti et al.]

cutaneous leishmaniasis. A biopsy was performed. Subsequent histopathology confirmed the suspected dermoscopic diagnosis of CS, showing noncaseating epithelioid granulomas (Figure 3).

Blood tests showed a high level of angiotensin converting enzyme (ACE) and antinuclear antibody (ANA). The patient did not present with pulmonary involvement.

\section{Discussion}

Sarcoidosis is a multisystemic granulomatous disease of unknown causes. It may involve various tissues and organs, but the lungs, lymph nodes, eyes, and skin are commonly involved. CS is usually an early manifestation of the systemic disease. Specific cutaneous manifestations of sarcoidosis are highly polymorphous, including solitary or multiple, red, yellow-brown or purple macules or papules, plaques, infiltrations, annular lesions, and subcutaneous nodules [1-3].

In past years, specific dermoscopic patterns of a variety of inflammatory and infectious skin disorders have been described, including some commonly considered CS in differential diagnosis [4,5]. Pellicano et al [4] reported a retro-

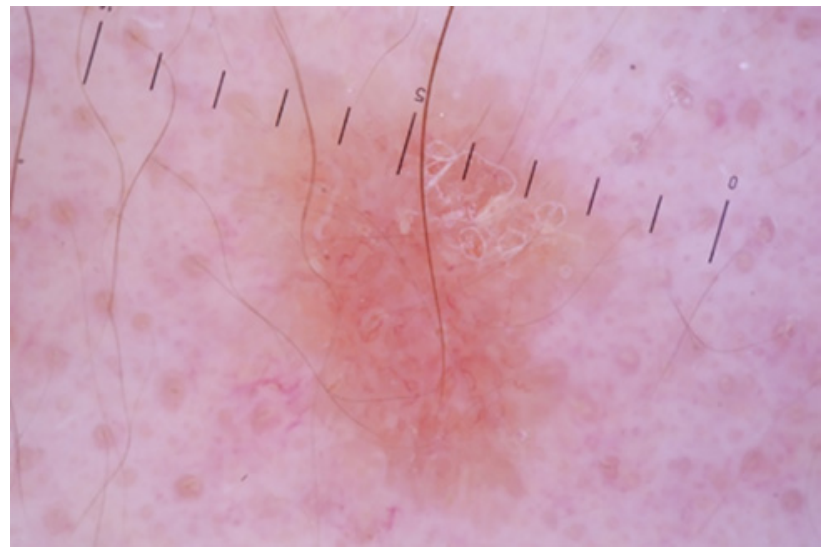

Figure 2. Dermoscopy (polarized $\times 20$ ) exhibits whitish-yellow scales and whitish structureless areas (red arrow) on a yelloworange background (black arrow), with diffuse linear irregular vessels. [Copyright: (O2018 Conforti et al.]

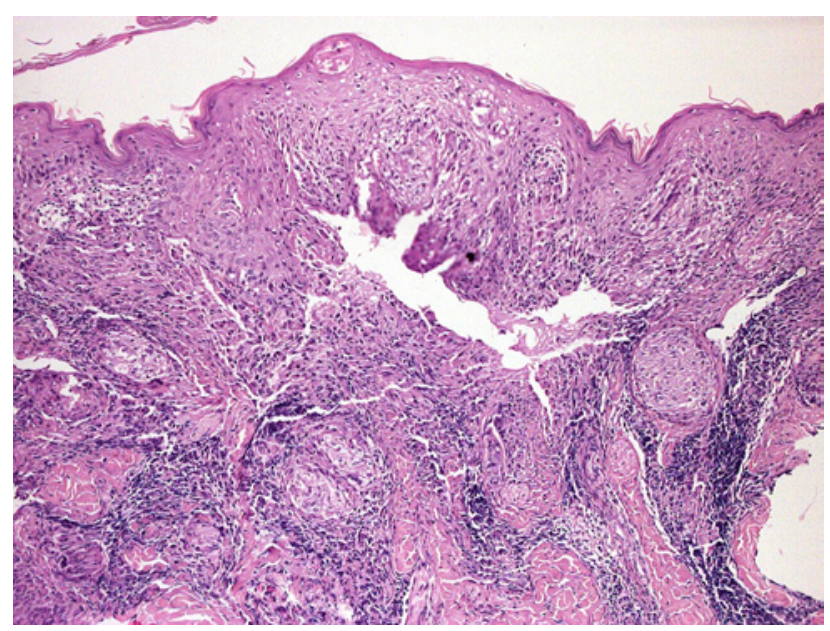

Figure 3. Histology $(\times 40)$ shows noncaseating epithelioid granulomas. [Copyright: @2018 Conforti et al.]

spective dermoscopic evaluation of the 7 cases of histologically confirmed CS. Linear vessels overlying the translucent orange ovoid structures were the only vascular structures seen in all cases [4]. In line with this, Vázquez-López et al evaluated the dermoscopic patterns in a large series of different nontumoral dermatoses; their series included 3 cases of CS, of which 2 lesions exhibited monomorphous linear vessels [5]. We also found linear vessels as the only vascular pattern in our case.

Histologically, CS shows noncaseating epithelioid granulomas, with minimal or absent lymphocytes or plasma cells (naked granuloma) [6]. Inside giant cells, Schaumann bodies and asteroid bodies may be found, although they are not specific. Many of these features are not exclusively seen in CS and may also occur in infectious diseases, immunologic/ foreign body granulomas, neoplasia, immunodeficiency disorders, and drug eruptions. For this reason, sarcoidosis is one of the "great imitators" clinically and histopathologically, though its diagnosis is facilitated by dermoscopy. 


\section{Conclusions}

In our patient, clinical features were indeed consistent with the diagnosis of AK. However, dermoscopy exhibited whitish structureless areas on a yellow-orange background associated with linear vessels, compatible with granulomatous skin disease. The dermoscopy findings changed the diagnosis from tumoral to granulomatous disease and led to the correct management.

\section{References}

1. Tchernev G. Cutaneous sarcoidosis: the "great imitator": etiopathogenesis, morphology, differential diagnosis, and clinical management. Am J Clin Dermatol. 2006;7(6):375-382. doi: 10.2165/00128071-200607060-00006.

2. Elgart ML. Cutaneous sarcoidosis: definitions and types of lesions. Clin Dermatol. 1986;4(4):35-45. doi: 10.1016/0738081X(86)90032-5.

3. Fernandez-Faith E, McDonnell J. Cutaneous sarcoidosis: differential diagnosis. Clin Dermatol. 2007;25(3):276-287. doi: 10.1016/j. clindermatol.2007.03.004.

4. Pellicano R, Tiodorovic-Zivkovic D, Gourhant JY, et al. Dermoscopy of cutaneous sarcoidosis. Dermatology. 2010;221(1):51-54. doi: 10.1159/000284584.

5. Vázquez-López F, Kreusch J, Marghoob AA. Dermoscopic semiology: further insights into vascular features by screening a large spectrum of nontumoral skin lesions. $\mathrm{Br} J$ Dermatol. 2004;150(2):226-231. doi: 10.1111/j.1365-2133.2004.05753.x.

6. Wilson NJ, King CM. Cutaneous sarcoidosis. Postgrad Med J. 1998;74(877):649-652. doi: 10.1136/pgmj.74.877.649. 